University of the Pacific

Scholarly Commons

University of the Pacific Theses and

Dissertations

Graduate School

1976

\title{
Increasing compliance to a medical regimen with a juvenile diabetic
}

Kathleen Lowe

University of the Pacific

Follow this and additional works at: https://scholarlycommons.pacific.edu/uop_etds

Part of the Psychology Commons

\section{Recommended Citation}

Lowe, Kathleen. (1976). Increasing compliance to a medical regimen with a juvenile diabetic. University of the Pacific, Thesis. https://scholarlycommons.pacific.edu/uop_etds/1920

This Thesis is brought to you for free and open access by the Graduate School at Scholarly Commons. It has been accepted for inclusion in University of the Pacific Theses and Dissertations by an authorized administrator of Scholarly Commons. For more information, please contact mgibney@pacific.edu. 
INCREASING COMPLIANCE TO" A MEDICAL REGIMUN

WITH A JUVENILE DIABETTC

\author{
A Thesis \\ Presented to \\ the Faculty of the Department of Psychology \\ University of the Pacific
}

In Partial Fulfillment

of the Requirements for the Degree

Maste; of Arts

by

Kathleen Lowe

May 1976 
This thesis, written and submitted by

Kathleen Lowe

is approved for recommendation to the Committee on Graduate Studies, University of the Pacific.
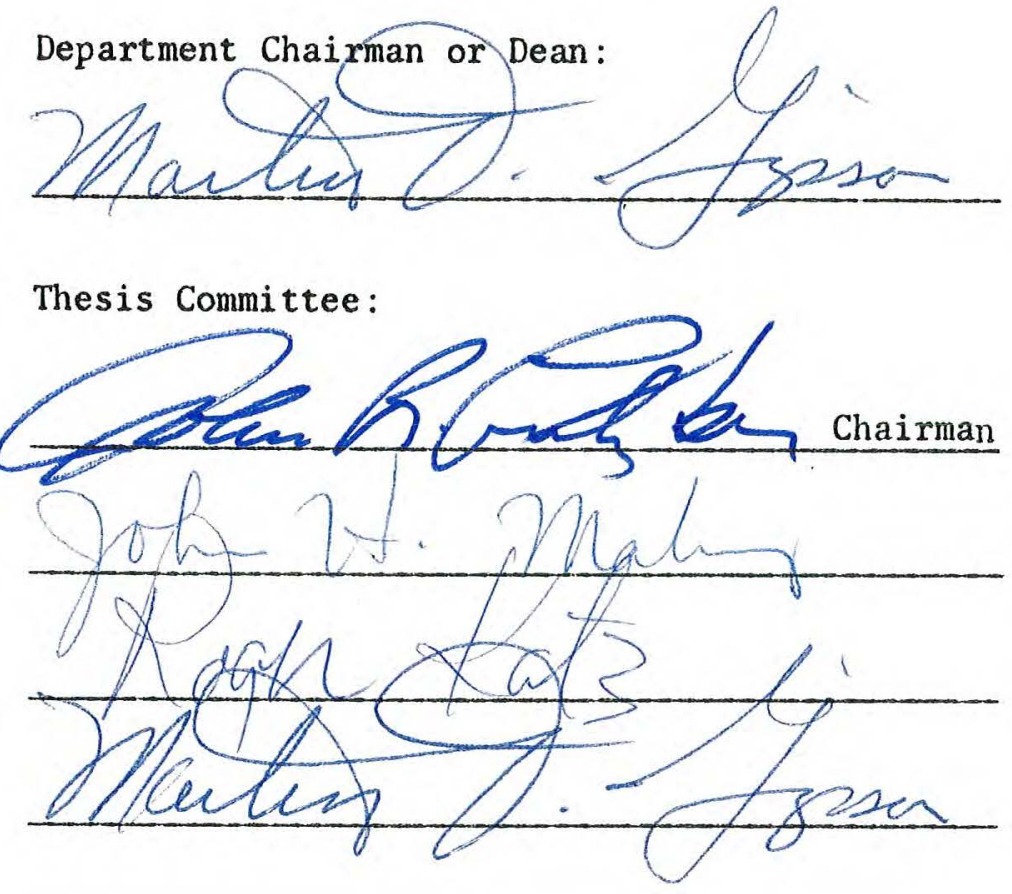

Dated $5 / 24 / 26$ 


\section{ACKNOWLEDGEMENTS}

I am particularly grateful to my committee chairperson, John Lutzkex, for his patience, prodding, and excitement. It was John who led me to develop my interest in applied behavior analysis and I have never regreted it. I wish to express my appreciation to thesis comittee members Hartin Gipson, Roger Katz, and John Mabry for their thoughtful criticism ana assistance throughout the study. I also wish to thank Judy Viventi and Pat Dixon for opening up the medical world to me and teaching me so much about diabetes. Thanks are finslly due to Gilbert Hermosillo for his encouragement and conscientious editing. 
Studies investigating the use of behavioral techniques in the treatment of health-related problems have recenty received considerable emphasis. Problens such as enuresis (Atthowe, 1972; Nordquist, 1971), obesity (Stuart, 1967), alcoholism (Miller, 1972; Sobell and Sobell, 1973), chronic gain (Fordyce, Fowler, Lehman, and DeLateur, 1975), and asthma (Neisworth, 1972; Renne and Creer, 1976) have been treated by the use of behavioral techniques.

Katz and Zlutnick (1975) mention two critical areas in which behavior analysis is particularly applicable to heaith care: a) rehabilitation, and b) patjent management. Rehabilitation involves learning behaviors related to specific physical disabilities, for example stroke patients relearning walking skills, and amputees learning various seif-care and vocational skilis. Patient maragement involves increasing or decreasing specific patient behaviors to ensure they follow prescribed treatment plans. Patients with chronic diseases such as diabetes or multiple - sclerosis mut often be placed on stringeit medical regimens. Thus, conpliance with physicians! requests to take medication, follow diets: or engage in exercise is a vital component of patient management and must be maintained if a patient is to sustain optimal health. In view of these considerations, knowledge of the conditions under which 
leaning best occurs, and the variables that control behaviow, might well facilitate patient recovery and make rehabilitation and patient-management programs more successful.

Patient noncompliance with a medical regimen is a typical problem confronting the medical profession. Physicians suspect a $50 \%$ noncompliance rate with prescribed regimens (Gillum and Barsky, 1974). Studies have shown that there is little or no association between compliance behavior and age, sex, socioeconomic status, and education of the patient (Marston, 1970). Furthermore, recent studies have shown that the acquisition of knowledge concerning illness and its treatment does not increase compliance. However, as the complexity of the medical regimen increases there is a marked decrease in compliance (Gillum and Barsky, 1974). These findings do not seem surprising in view of the fact that adopting new medical regimens means adopting entirely new patterns of behavior. Contingency management procedures may be necessary to maintain patient compliance with these regimens (Irvis, 1976).

Sand, Trieschmann, Fordyce, and Fowler (1970) conducted several studies in which behavioral techniques were used to decrease patient noncompliance in a rehabilitation setting. One study involved a 28 year old, hospitalized, male paraplegic. The subject was expected to complete a number of seli-care tasks including catheter irrigation, 
maintenarce of scheduled medication intake, personal oathing and dressing, and putting on his braces. The patient often neglected to complete many of these tasks, or took so long to do them that he missed his scheduled occupational and physical therapy sessions. Treatment consisted of giving the subject a list of behaviors he was to complete on a daily basis. Introduction of the ljst produced improved performance; at the end of the first week the subject performed from 13-22 of the behaviors, with 22 as the total possible. Next, weekly quotas of the prescribed behaviors were set, increasing by one each week. If the subject met his quotas he was rewarded with a weekend home pass. At the end of the first week of this condition the subject was consistently performing 19 out of the 22 behaviors. This high rate of compliance was maintained for the following 4 weeks until discharge, after which the patient continued to engage in self-care behaviors.

There are several possible reasons why noncompliance with health care procedures is so predominant. The first is that physicians usually give verbal instructions - or pamphlets to their patients and expect them to carry on at home without any further contingencies. Many studies have shown that instructions, written or verbal, are not sufficient to change behavior (Hursh, Schumaker, Fawcett, and Sherman, 1973; Iutzker and Drake, 1976; Quititch, 1975). 
Miller, Hersen, and Eisler (1974) conducted a study which looised at the effectiveness of instructions, written agreements, and reinforcement to help alcoholics decrease their rate and amount of drinking. The results showed that instructions and signed agreements had a linited influence on drinking, but those receiving rejnforcement for compliance significantly decreased drinising. A second probable cause of noncompliance is that of narrow stimulus control. The patient may indeed learn how to perform his required medical behaviors in the hospital or physician's office, but this is no guarantee they will generalize outside the office to the patient's home. Bebavioral changes in one setting are usually specific to that setting and will not generalize to another setting unless the same contingencies operate in the other setting(s) (Baer and Wolf, 1967).

One way to deal with the issue of stimulus control would be to train a patient or his parents to control his own behavior in the natural environment. Studies have shown that parents can be effective behavior change agents, using contingencies readily availabie in the home setting (Johnson and Katz, 1973). Phillips (1968) has shown that an effective way for parents to increase self-care, and other behaviors, is by utilizing a token economy or point system in the homer.

Diabetes is a prevalent disease involving complex 
self-care procedures. It is a chronic, inherited condition in children which prevents the body from using sugar nornally. Approximately $5,000,000$ people or 1 in every 40 persons have diabetes (Getting Started At Home: Practical Information for Learning to Live with Diabetes, 1975 ). There is no cure for this disease, but it can be controlled by maintaining a proper baiance between diet, exercise, and insulin injections, The physician typically prescribes a strict regimen which the diabetic must follow to miantain a desirable level of health. Behaviorally, this involves learning and maintaining a new set of behaviors which include insulin inoculation and urine testing. Along with that, old behaviors must be changed which, in this case, are prinarily exercise and eating habits.

To the author's knowledge there have been no published studies investigating the use of behaviorai techniques in the manageneat of diabetes. The purpose of this study was to examine the effects of positive reinforcement (in the form of a point system) on compliance to a medical regimen with a juvenile diabetic. An additional aspect of this study was that compliance was required in the subject's home, with a gradual shifting of responsibility for the contingencies from the experimenter to the subject's mother.

\section{Method}


pediatxician in response to a letter asking for noncompliant jwenile diabetice, served as the subject.

The child was born with cystic fibrosis and was diagnosed as diabetjc in March, 1975. Since her diagnosis she had been hospitalized 4 times due to diabetic acidosis, a toxic condition resulting from the bodily's inability to utilize sugar.

The child was entirely dependent on her mother fo: the care she required. The mother administered the child's daily insulin injections, tested her urine, planned and served a special diet, and maintained her general skin care.

During an initial interview with the subject's mother she corroborated the physician's report of her daughter's noncompliance. The mother voiced her concern about the complexity of her daughter's care and increasing discomfort. She welcomed assistance and was eager to hear the details of the program.

Setting

All observations and interventions took place in the subject's home which was located in a rural trailer court in Modesto, California.

\section{Behaviozal Measures}

The dependent variable was the percentage of intervals the subject was in compliance with three medical responsibilities: a) urine sampling, b) diet, and c) footcare, 
all of which had to be met for her to maintain optimal health. Compliance was defined as the completion of a prescribed medical task. That is, if the subject followed an instruction to engage in a self-care behavior at the prescribed time, or within 15 minutes before or after that time. it was scored as a "yes" under compliance. If she did not comply with the instruction within the time allotted, the subject's mother reminded her to complete the task, and it was scored as a "no" under compliance.

There were two independent variables: (a) memo, and (b) a point system. In the former condition the medical instructions were written in memo form and given to the subject by the experimenter (Quilitch, 1975). The memo was similar to the type of instructions a physician would give his patients (see Appendix B). The memos described ard explained appropriate self-care behaviors for the subject, and Iister the times they were to be completed. (This is discussed in more detaij later.) In the second condition, a point system, the subject earned points for complying with the inedical instructions. These points could iater be exchanged for daily and weekly reinforcers. (See Procedure.)

The data for the study were recorded by the subject's mother on charts provided by the experimenter. The mother was expected to chart the results of each urine test and the time it was given, the time and content of the subject's meals, and to iist the foot-care behaviors that were completed by the subject dailg. The mother did not prompt 
any behaviors, but if the subject omitted a task, the mother toid her to complete it after waiting 15 minutes. The subject's behavior was then scored as noncompliant. The cliarts were not made accessible to the subject until the point-aystem condition. The experimenter stressed to the mother the importance of following the above rules, and the experimenter and mother practiced the procedures several times before actual data collection began. Procedures for obtaining reliability measurements have been extremely limited and sometimes inappropriate in home based treatments (Kazdin, 1974). This is often be.cause the presence of the experimenter may have a reactive effect on the behavioxs of the subject. That is, if the subject is aware that he is being observed he may alter his behavior in response to that observation instead of to treatment. In this study reliability was evaluated by comparing data collected by the subject's mother and her older sister. Other data were collected by the experimenter to substantiate if, in fact, the subject's mother was recording data accurately. This was accomplished in the following marner.

The mother tested the subject's urine daily and recorded the results. In addition, she was instructed to save one-half of every urine sample she collected. The experimentex then tested them separately to corroborate the data on the charts. The subject's mother also observed and 
charted the subject's foot-care behaviors. These behaviors included washing her feet every night before bed and changing her socks each morning. The subject was given seven washcloths for use every day of the week. The experimenter checlsed the laundry for dirty socks and washoloths on random, unannounced visits throughout the study. The subject's sister was instructed to independently chart the subject's meals and snacks several times a week to provide a reliability measure on the diet. In addition, she independently charted the subject's compliance with urine testing and foot-care once during each condition.

Reliability was calculated by dividing the number of agreements by the total number of agreements plus disagreements $x$ 100. Reliability between the subject's mother and sister: averaged 98\% agreement for foot-care behaviors, 95\% for urine testing, and $87 \%$ for the diet. Reliability between the experimenter and the mother's reports averaged $81 \%$ agreement on footcare and $100 \%$ agreement on urine testing.

Design and Procedures

A multiple baseline across three medical responsibilities, foot-care, urine testing, and diet, was used (Baer, Wolf, and Risley, 1968). To meet the responsibilities for uriae testing the child collected four urine samples per day, before breakfast, before lunch, betore dinner, and before bedtime. The child tested for concentration 
of glucose in the urine and for acetone (a byproduct of the metabolism of fat). To meet responsibilities for the diet the child was required to follow a prescribed diet, eat only those foods listed and eat them at the prescribed times. To meet foot-care responsibilities the child was required to majntain a pattern of daily foot-care.

Prior to data collection the experimenter met with the subject's pediatrician and family to review hex particular medical regimen and the problems she was having in complying with the physician's instructions.

Next, a pre-test was given to ascertain if the subject could perform the behaviors involved in the medical instructions (see Appendix A). This was to ensure that the subject's noncompliance was not due to her lack of knowledge of how to perform the requisite behaviors. The experimenter simply asked the subject to test her urine and wash and inspect her feet. The experimenter gave the subject a short oral quiz on her diet, covering what foods she could and could not eat, when she should eat, and how much. The experimenter then taught the subject any behaviors she did not perform appropriately. This involved the use of modeline the correct responses, then asking the subject to rehearse them until she could perform them correctly by herselt. The experimenter explained the diet to the subjeet and Iisted for her the foods she could and could not eat. 
The experimenter then met with the subject's mother, gave her the charts, instructed her on the procedures of data collection, and instructed her to begin baseline (see Appendix C). She recorded the subject's degree of compliance on all three instructions for six days.

Following baseline, written instructions in the form of a memo were given to the subject on the first behavior, foot-care. The memo was handed to her at the beginring of the condition, and she was asked to read it carefully to the experimenter. The subject ther posted it in her bathroom. Memos were given for the second and third behaviors in a staggered fashion over time. Since the memo was efiective for the diet, it was reintroduced a second time for the other two behaviors in order to determine if one more application would produce a change. The subject's mother continued to record compliance, which was not rewarded if it occurred.

The final condition was the point system, which was also introduced in a staggered fashion over time. The experimenter explained to the subject that she could earn . points by following the instructions she had been given. For example, she could earn points every time she collected her urine and tested it ( 8 points daily), for correct dietary procedures ( 5 points daily), and for engaging in proper foot-care behaviors (4 points daily). The subject traded her points for daily and weekly reinforcers. The mother was asked to post the data where the subject could 
see them, and the experimenter visited the home two or three times a week to comment on her progress. The subject was not exposed to the point system for correct dietary procedures because the memo appeared to be effective in increasing compliance, (Sce Results section.) Therefore, she received points for urine testing and footcare only. She was required to earn a total of 12 points to earn her daily reinforcer and a total of 70 points to earn her weekly reinforcer. The subject exchanged her points for dietetic sodas, diabetic treats, bike riding, games, movies, and special trips. The subject's mother delivered the daily reinforcers and the experimenter delivered the weelly contingencies for approximately one month, At the end of the month the subject's parents began to deliver the weekly contingencjes and the experimeter faded her involvement to weekly phone calls, letters, and home visits every three weeks.

\section{Results}

Figure 1 shows the percentage of intervals in which compliance occurred. Compliance was calculated by dividing the number of times the subject performed the required behaviors by the total number of responsibilities completed that day. The results indicate that the point system was effective in increasing compliance with foot-care and urine testing responsibilities. A memo, which was relatively 
ineffective in increasing foot-care and urine testing, appeared to facilitate proper dietary behaviors.

\section{Insert Figure 1 about here}

As can be seen in Figure 1, the average percentage of compliance for foot-care during the baseline and memo conditions was $72 \%$. Compliance increased to $1.00 \%$ when the point syster was instituted and remained stable thereafter.

During baseline, compliance to urine testing was sporadic, varying, from 0 to $50 \%(\bar{x}=19 \%)$. Compiiance during the memo condition was also inconsistant, the mean increasing to $35 \%$. When the point system was instituted, compliance increased and the subject tested her urine consistentiy with the exception of one occasion $(\bar{x}=97 \%)$.

The data on compliance to diet show that during baseine the subject complied approximately $72 \%$ of the time, again with considerable variability. When the memo was introduced the subject's compliance to diet increased and was maintained at a consistently high level throughout the remainder of the study $(\bar{x}=99 \%)$.

Since the memo appeared to be effective in maintaining compliance to diet, the point system was not introduced.

Data collected on the three-week follow-up indicate that compliance to all three medical responsibilities was matrained. 


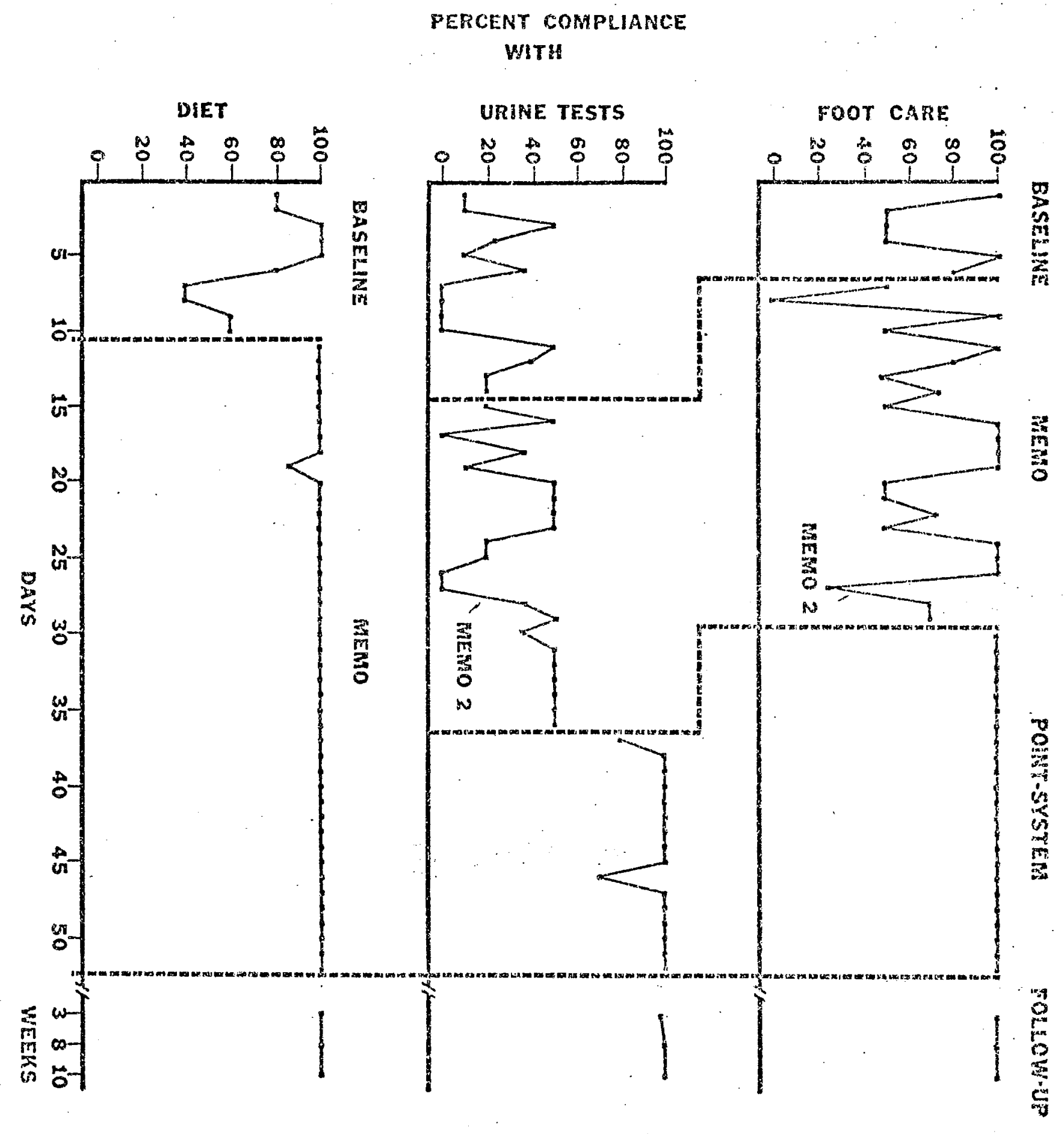

Figure 1. Compliance behaviors with three medical responsibilities. 


\section{Discussion}

The results indicate that a point system was effective in increasing compliance to a medical regimen with a juvenile diabetic. A memo increased compliance to diet. Follow-up data suggest that the subject's mother carried out the procedures successfully after the experimenter had discontinued her involvement. That is, all three medical responsibilities were maintained. In addition, both the subject and her mother expressed satisfaction with the resul.ts. The subject reported that she felt healthier more of the time and looked forward to her weekly rewards. The subject's mother reported that her child was engaging in her self-care activities independently and without having to be reminded to perform them.

The study supports the results of other investigators (see Katz and Zlutnjck, 1975) and show that contingent positive reinforcement can be used to increase compliance with medical regimens. These results have important implications for diabetics and other chronically ill patients, as well as for physicians, nurses, and other health care personnel, because compliance not only increased, but remained at a consistentiy high level when a relatively simple behavioral intervention was employed.

In diabetes, consistency to routine is critical. Diabetes can be controiled by a careful balance of insulin, diet, and exercise. Urine tests are a diabetic's means of assessing this balance. Results of urine tests give a 
diabetic feedback that allows him/her to rearrange sugar intsise, change exercise schedules, or readjust insulin. If testing is done only occasionally, the diabetic runs the risk of serious complications. Careful, consistent monitoring of urine tests allows her/him to have better control over the disease, thus allowing better health and less frequent hospitalization.

Training in the principles and procedures of behavior manasement could be one way for physicians to tackle the frequent problem of patient noncompliance to medical regimens. Physicians could continue to inform the patients on what procedures they should follow to maintain good health. Physicians could follow this step up by teaching nurses or paraprofessionajs to create appropriate behavior management programs with patients. The staff could then monitor the patients in their homes, eventually transferring the progran responsibility to patients themseives, their parents, or other family members.

The purpose of the memo condition in this study was to simulate the usual medical practice of giving the patient written instructions on how to maintain his/ler medical regimen; physicians may give verbal instructions in the office, then supply patients with additional 1iterature to read at their leisure. Fhysicians have no way of knowing if their patients read this literature. This study diflers somewhat in that the child wos asked to read 
the writen instructions out loud jin the presence of the experimentew, and to post the memo on her bathroom or

kitchen wal1. Studies by Miller et al. (1974) and Quilitch (1975) have shown that memos or written instructions alone are usually not effective in producing durable bekavior change. This was the case for the first two medical responsibilities in the current study. However, the memo appeared to be effective in increasing and maintaining compliance to the diet. Compliance to the diet may not have been as difficult as it obviously was to urine testing and foot-care. The subject had to learn to eat at scheduled times, and to eat all of the foot on her plate, but the content of ber diet was not stringent. She was allowed a limited intake of sweets so was not deprived of any favorite foods. Thus, the subject had to regulate har eating habits and not learn entirely new behaviors as was the case for the other two responsibilities. Compliance to a diet needs to be investigated in the future to see if it is affected by the memo in the same way, although much of the literature on obesity suggests the contrary. A physician could always try the memo first, requiring the patient to read the instructions, then switch to a point system if needed. A point system to increase compliance is easily effected and inexpensive in terms of both the physieian's time and patient's money. 
Not answered by the current study was whether or not the posting of results alone would have increased conpliance by the subject. During the point system, the subject's mother posted the child's data sheets so the child could monitor her progress if she wished. However, she was not instructed to do so. Feedback was available if the child did look at her charts and could have been confounder with the point system. It is not known if the point system alone or the feedback and point system were effective in increasing compliance.

Replication of these resujts would substantiate the notion that simple behavioral procedures can be used to increase compliance to medical regimens (and thereby better health) with chronically ill patients. 


\section{REFERENCES}

At thowe, J. M. Controlling nocturnal enuresis in severely disabled and chronic patients. Behavior Therapy, $1972,3,232-239$.

Baer, D. M, and Wolf, M. M. The entry into natural communities of reinforcement. Ir R. Ulrich, $\mathrm{T}$. Stachnik, and J. Mabry (Eds.), Control of Human Behavior: From Cure to Prevention. GIenview, III: Scott, Foresman, and $\mathrm{Co}, 1970$.

Baer, D. M., Wolf, M. M., Risely, T. R. Some current dimensions of applied behavior analysis. Journal of Applied Behavior Analysis, 2968, I, 91-97.

Fordyce, W. E., Fowler, R. S., Lehman, J. F., and DeLateur, B. J. Some implications of learning in problems of chronic pain. In R. Katz and S. Zlutnick (Eds.), Beharior Therapy and Health Care: Principles and Applications. Fergamon Pxess, inc., 1975.

Getting Started At Home: Practical Information for Learning to Live with Diabetes. Brecton, Dickinson and Co., New Jersey, 1975 .

Gillum, R. F., and Barsky, A. J. Diagnosis and managenent of patient noncompliance. Journal of the American Medical Association, 1974, I2, 1563-1567.

Hursh, D, E., Schumaker, J. B., Fawcett, S. B., and Sherman, J. A. A comparison of written and direci instructional. methods. Paper presented at the American Dsychological Association, Montreal, Quebec, Canada. August, 1973.

- Irvin, W. The effects of facilitative packaging and verbal reinforcement ipon compliance with medical regimens by psychiatric cutpatients. Unpublished master's thesis, University of the Pacific, 1976.

Johnsor, C.A., and Katz, R. C. Using parents as change agents for their children: a review. Journal of Child Psychology ard Psychiatry, 1973, 14, $18 \overline{\mathrm{I}-200 .}$

Katz, R. C, and Ziutnick, S. Behavior Therapy and Health Care: Principles and Applications. Pergamon press Inc. 1975 . 
Kazdin, A, E. Self-monitoring and behavior change. In M. J. Mahoney and C, E. Thoresen (Eds.), Self-Control: Power to the person. Monterey: Brooks/Cole, 1974, $218-246$.

Lutzkex, J. R., and Drake, J. A comparison of trainertraining techniques to produce rapid toilet training. To be presented at the convention of the American psychological Association, Washington, D. C., September, 1976.

Marston, M. Compliance with medical regimens: A review of the 1iterature. Nursing Research, 1970, 19, 312323.

Miller, P. M. The use of behavioral contracting in the treatment of a.lcoholism: A case report. Behavior Therapy, 1972, 3, 593-596.

Miller, P. M. Hersen, M., Eisler, R. Relative effectiveness of instructions, agreements, and reinforcement in behavioral contracts with alcoholics. Journal of Abnormal Psychology, 1974, 83, 19-24.

Neisworth, J. T. Operant treatment of asthmatic responding witi the parent as therapist. Behavior Therapy, $1972, \underline{3}, 95-99$.

Nordquist, V. M. The modification of a child's enuresis: Some response-response relationships. Journal of Applied Behavior Analysis. 1971, 4, 357-365.

Phillips, E. L. Achievemert Place: token reinforcenent procedures in a home-style rehabilitation setting for "pre-delinquent" boys. Journal of Applied Behaviors Analysis, 1968, $1,2 \overline{13-224 .}$

Renne, C. M., and Creer, T. L. Training children with asthma to use inhalation therapy equipment. Journal of Applied Behavior Analysis, 1976, 9, 1-11.

Quilitch, R. H. A comparison of three staff management: procedures. Journal of Applied Behavior Analysis, 1975, 8, $59-66$.

Sanc, D. L., Frieschmann, R. B., Fordyce, W. E., and Fow?ex: R. S. Behavior modification in the medical rehabilitation setting: Rationale and some applications. In R. Katz and 8 . Zlutnick (Eds.), Behavion Therapy and Health Care: Principles and Applications. Pergamon Press, Inc., 1975. 
Sobel1, M. B. and Sobel], I. C. Individualized behavior therapy for alcoholics. Behavior Therapy, 1973, 4, $49-71$.

Stuart, R. B. Behavioral control of overeating. Behavior Research and Therapy, $1967,5,357-365$. 
APPENDIX A 


\section{Pre-test}

1. Demonstrate how you would test youx urjne for glucose. Did the subject complete the following steps:
a. Save urine in a cup.
yes no
b. Dip tes-tape into urine.
yes no
c. Wait $10 \mathrm{sec}$, and compare results with color chart.
yes no

2. Demonstrate how you would test your urine for acetone. Did the subject complete the following steps:
a. Draw up part of urine specimen in eye dropper. yes no
b. Place 1 drop of urine on acetest tablet.
yes no
c. Wait 10 sec. and compare results with color chart.
yes no

3. Demonstrate how to wash and inspect your feet. Did the subject complete the following steps:

a. Wash feet carefully with lukewarm water.

yes no

b. Pat (not rub) feet dry. yes no

c. Spread toes and pat dry between toes. yes no

d. Inspect entire feet for corns, cuts, bruises, callouses, etc. jes no 


$$
\text { Pre-test for Diet }
$$

1. The food exchange system means you can:
a. safely eat all foods on each list without measuring or weighing them.
b. eat any type of food as long as you feel well.
c. interchange foods within the same food group to add variety to your diet.

\section{True or False}

1. It is safe to skip a meal once in a while.

2. It's okay to have a candy bax whenever you feel like it.

3. If you can't eat all of your meal, save it for a snack later.

4. You can't have too much sugar on the exchange list.

5. You must try to eat all foods planned for each meal.

6. I can drink aII the diet soda I want without it upsetting my diet.

7. Give an example of a healthy breakfast, lunch, and dinner.

8. What do you do when you have just been execcising and you suddenly feel weak, dizzy, shaky, and hungry?

9. Demonstrate how to use the exchange list. 
APPENDIX $B$

25 
MRMO ON FOOT CARE

People with diabetes have very sensitive feet so they should take very good care of them to avoid infections. Dadiy goot inspections and washings should help you to avoid soot pioblems.

What to do.

1. Wear clean, colorfast socks. If your feet become wet, for any reason and the color of your socks rubs off on them, do not weax them anynore.

2. Put on a fresh, clear pair of socks every morning.

3. Wash your feet carefully everyday. Bathe your feet in warm, not hot, water. Use mild soap and the soft washcloths provided.

4. Pat your feet dry (don't rub!) with a soft, clean towel. Be sure and spread every toe and pat dry between your toes very gentiy.

5. Inspect your feet for cuts, sores, patches or hard skin, or cracks in your skin after you wash your feet. If you have any of these tell your mother. Do not try to put any medicine on your feet yourself. 
MEMO ON URINE TESTS

Urine tests help you to know how well your body is using the sugar you get from the lood you eat. You must test your urine 4 times a day to know how healthy you are.

You should test your urine before breakiast, before Iunch, before supper, and before bedtime.

How to test your urine.

When you get up in the morning, urinate, then drink a glass of water. Do not test this urine because it has been stored in your bladder. Test the next urine sample.

1. Urinate before oreakfast and save your urine in a clean bottle.

2. Test your urine for sugar.

a. Withdraw about $1 \frac{1}{2}$ inches of Tes-Tape.

b. Dip the Tes-Tape into the urine, remove, and wait. I minute. If the tape stays yellow your urine does not have any sugar in it.

c. Compare the darkest area of your tape with the TesTape color chart. If the tape matches the color with $\frac{1}{2} \%$ or higher, wait another minute and corpare the tape with the color chart again.

3. Test your urine for acetone.

a. Place 1 Acetest tablet on a piece of white paper.

b. Put 1 drop of urine on the tablet.

c. Wajt 30 seconds then compare the color of the tablet with the Acetest color chart. 
4. If your urine is $\frac{1}{2} \%$ or 3 ttt or higher, and your Acetest is moderate or above, your body isn't using sugar very well and your mother should call the doctor.

5. Follow these procedures before every meal and bedtime. 
MEMO ON DIET

Your diet gives you energy and keeps you healthy?

People with diabetes must eat about the same amount of food everyday, and eat about the same time everyday. This is to make sure that your insulin works best for you.

Your mother will pian your diet and you must eat what she gives you. Always eat at the planned time. If. you are hungry for something special, do not sneak it, tell your mother and she can work it into your diet.

What to do:

1. Eat breakfast right after your insulin shot in the morning.

2. Have a snack around 10:30.

3. Eat lunch around noon.

4. Have a snack around 3:00.

5. Have dinner with your family at your regular time.

6. NEVER SKIP OR DELAY A MEAL!:

7. Try to eat everything on your plate.

8. No sneaking extra snaciss! 
APPENDIX C 


\begin{tabular}{|c|c|c|c|c|c|}
\hline $\begin{array}{l}\text { 1. Put on clean, colorfast } \\
\text { socks this morning when } \\
\text { dressing. }\end{array}$ & & & & & \\
\hline $\begin{array}{l}\text { 3. Pat your feet dry. } \\
\text { Spread your toes and } \\
\text { par dry between each } \\
\text { toe. }\end{array}$ & & & & & \\
\hline $\begin{array}{l}\text { 5. Bonus points: Cut your } \\
\text { toenails when needed. Cut } \\
\text { them straight across the } \\
\text { top of the nail, never } \\
\text { shorter than the tops of } \\
\text { the toes. }\end{array}$ & & & & & \\
\hline $\begin{array}{l}\text { TOTAL POINTS EARNED DAILY } \\
\text { Each "yes" equals } 1 \text { point. } \\
\text { Each "no" equais o point. }\end{array}$ & & & & & \\
\hline
\end{tabular}


Urine Tests

Results of Urine and Acetests

Urine Tests

Compliance with Urine and Acetests (mark yes or no)

$\begin{array}{clll}\text { Urine Test } & \text { Total pts. } \\ \begin{array}{l}\text { before } \\ \text { breakfast lunch }\end{array} & \text { "yes" - } \\ \text { lunner bed-time } 1 \text { point }\end{array}$


Date

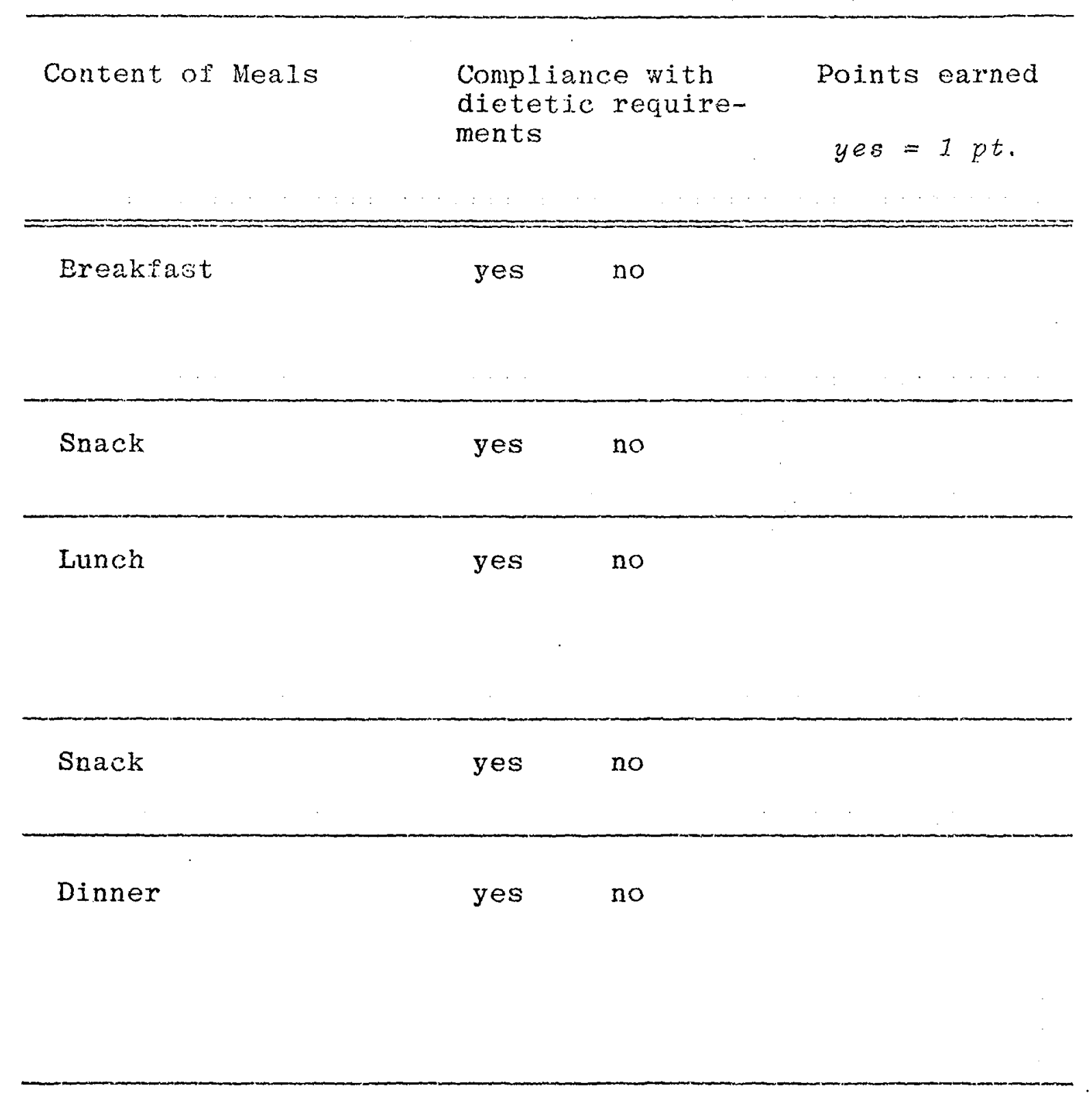

